\title{
Role of Coronary Artery Bypass Surgery in Acute Myocardial Infarction
}

\author{
William Y. Shi and Julian A. Smith
}

\subsection{Introduction}

Coronary artery bypass grafting (CABG) is one of the most commonly performed procedures worldwide. Its place in the treatment of coronary artery disease has been established for decades with the benefits of $\mathrm{CABG}$ versus percutaneous coronary intervention (PCI) in various scenarios being extensively investigated. Recent major landmark randomised clinical trials such as SYNTAX, ${ }^{1} \mathrm{EXCEL}^{2}$ and $\mathrm{NOBLE}^{3}$ have helped to define the patients in which each approach is likely to be most successful. Indeed, the last decade has seen most centres embrace the "Heart Team" concept, whereby a collaborative approach helps to optimise patients' outcomes.

The benefit of timely revascularisation in acute myocardial infarction (MI) is well established. The role of PCI and thrombolysis is discussed elsewhere; however, the former has now become the preferred strategy worldwide and is associated with

\footnotetext{
${ }^{1}$ Mohr FW et al., Lancet 2013.

${ }^{2}$ Stone GW et al., N Eng J Med 2016

${ }^{3}$ Makikallio T et al., Lancet 2016
}

\footnotetext{
W. Y. Shi

Department of Cardiothoracic Surgery, Monash Health, Melbourne, VIC, Australia

Faculty of Medicine, Melbourne Medical School, University of Melbourne, Melbourne, VIC, Australia

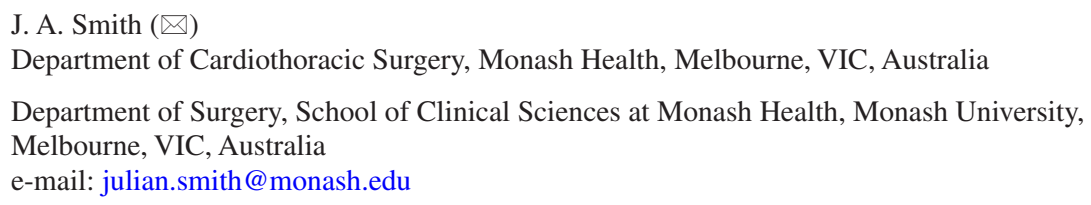


good outcomes. In general, PCI and thrombolysis can restore blood flow to the myocardium in the timeliest manner, whereas $\mathrm{CABG}$ is generally associated with a time delay and as such, is utilised in only around 5\% of acute MI cases in today's practice.

Historically, large multicentre randomised trials were designed to assess the efficacy of thrombolysis and PCI. CABG was never investigated as a primary treatment modality for STEMI in major randomised trials. Thus, high-level evidence for its place in the management of acute MI has never been firmly established. Practical, logistic and economic restraints mean that surgery remains, in most cases, a third option behind PCI and thrombolysis in the management acute MI. Nevertheless-as this chapter will discuss-CABG serves an important role in a select subgroup of patients.

\subsection{Indications for CABG in Acute MI}

\subsubsection{Unsuccessful PCI and Incomplete Revascularisation}

Patients with unsuccessful PCI, or those in which a less than satisfactory result has been achieved with PCI, should be referred for surgery if the coronary anatomy and patient factors are suitable. A substantial portion of patients with acute MI have multi-vessel coronary disease which may be long-standing and difficult to manage percutaneously.

Despite effective management of the culprit lesion via PCI, CABG may be required once the patient is stabilised in order to complete revascularisation of diseased territories not amenable to PCI. Studies have suggested reasonable early and late outcomes for those undergoing emergent CABG after failed angioplasty. Even in patients with cardiogenic shock post PCI, CABG may be associated with improved survival compared to post-PCI medical therapy alone.

Although uncommon, complications encountered during PCI for STEMI may necessitate emergency CABG. These include failed stent deployment, stent fracture, coronary artery dissection, coronary artery perforation or recurrent acute thrombosis. In these scenarios, patients with ongoing ischaemia will benefit from $\mathrm{CABG}$ so as to minimise further myocardial damage.

In general, restoration of blood flow should be a priority, and patients who continue to have clinical evidence of ongoing ischaemia post-PCI should be offered $\mathrm{CABG}$ if no further percutaneous options are available. The appropriateness of surgery differs on a case-by-case basis, and co-operation between cardiologists and cardiac surgeons is crucial.

\subsubsection{Mechanical Complications of Acute Myocardial Infarction}

Common mechanical complications of myocardial infarction are acute mitral regurgitation, rupture of the interventricular septum and rupture of the left ventricular (LV) free wall. These patients almost always require emergency surgery to repair the defect.

While a culprit lesion may be addressed via PCI, the restoration of blood flow will not reduce the haemodynamic burden of the mechanical defect. In select cases, it may 
be appropriate to perform PCI to the culprit lesion-if it can be done so in a timely manner-and then subsequently transfer the patient to the operating theatre for repair of the mechanical defect and grafting of other diseased vessels not addressed via PCI. In general, this approach is unsuitable in patients with rupture of the LV or ventricular septal defect as in these cases, the infarcted area cannot be salvaged with PCI and repair of the defect and restoration of normal haemodynamics is of higher priority. In the operating theatre, grafting of the culprit vessel in those with LV rupture or ventricular septal defect (VSD) is generally not performed due to absence of viable myocardium.

Surgery for mechanical complications of myocardial infarction is associated with a high early mortality risk, ranging between 30 and 50\%. Predictors of death are older age, worsening renal function, emergency surgery and higher filling pressures. Infero-posterior ventricular septal rupture is associated with the worst prognosis, owing to a more technically challenging repair and a higher rate of right ventricular dysfunction. Five-year survival ranges between 40 and $60 \%$.

\subsubsection{CABG as the Primary Revascularisation Strategy in STEMI}

There are no randomised trials directly comparing CABG with PCI in the setting of acute MI. In the acute setting, PCI is most often the appropriate approach if there is a clear culprit lesion, the distal flow is poor, and a stent can be deployed safely and more rapidly than performing CABG. However, approximately $50 \%$ of patients presenting with acute MI will have multivessel disease, and in stabe patients with complex coronary anatomy and higher SYNTAX scores, CABG may be preferable to PCI given its superior longterm clinical results, as demonstrated from observational and randomised studies.

There are cases in acute MI where CABG may be preferable. Patients who no longer have signs of active ischaemia but exhibit complex coronary anatomy may be better suited for CABG. Clinical factors such as diabetes and poor ventricular function also represent indications for $\mathrm{CABG}$ over PCI, even in the acute setting. The decision on which modality to pursue should be individualised and may also relate to whether surgical facilities are available in the local setting.

The advantage of CABG - even in the acute scenario - is that complete revascularisation can be achieved, rather than only targeting the culprit lesion. Complete revascularisation restores myocardial perfusion and provides the best opportunity for myocardial salvage. The importance of complete revascularisation is even more pronounced in patients with multivessel disease, as myocardium may continue to be compromised even after a culprit lesion is addressed via PCI.

\subsection{Timing of CABG After Acute Myocardial Infarction}

The optimal timing of CABG after acute MI is controversial. In many cases, there are clear indications for emergency surgery. In situations of ongoing ischaemia, mechanical complications or severe cardiogenic shock, patients should be transferred directly to the operating theatre. However, in the absence of these, the ideal timing of surgery is less well established. 
While early surgical revascularisation may limit the size of the infarct and reduce the potential for mechanical sequelae, there is the risk of reperfusion injury associated with early restoration of blood flow. This may lead to haemorrhagic infarction, extension of the infarct and greater scar development. It has been postulated that early $\mathrm{CABG}$ - either with or without cardiopulmonary bypass - after MI may augment the systemic inflammatory response seen after STEMI.

In a multicentre study of 32,099 cases, Lee and colleagues ${ }^{4}$ showed that for transmural myocardial infarction, in-hospital mortality decreased with increasing time interval between myocardial infarct and surgery. Mortality was $14.2 \%$ for those undergoing surgery within $6 \mathrm{~h}$, and $2.7 \%$ for those having surgery beyond 15 days after presentation. Revascularisation within 3 days of MI was an independent predictor of in-hospital mortality on multivariable regression analyses.

Similarly, Thielmann et al. ${ }^{5}$ reported their results of 138 cases of CABG in acute MI and found an overall mortality rate of $8.7 \%$. The mortality rate however varied between $23.8 \%$ for those operated on between 7 and $24 \mathrm{~h}$ after presentation and $2.4 \%$ for those receiving surgery at 8-14 days post-presentation.

At our centre, we generally avoid CABG on patients presenting with an acute STEMI and prefer to wait a period of 3-5 days until surgery in the absence of absolute indications for emergent CABG. Indeed, in cases where early surgery is an absolute necessity, we are usually prepared to offer post-operative mechanical circulatory support if the patient had reasonable premorbid function, as mortality is likely to result from pump failure.

In addition to timing of surgery, risk factors for mortality in surgery for MI are increasing age, renal impairment, number of previous MIs, hypertension, re-operative surgery, cardiogenic shock, reduced left ventricular ejection fraction, the need for cardiopulmonary resuscitation, left main stem coronary disease and the need for intra-aortic counterpulsation. On the other hand, preserved left ventricular function, younger age, male gender and non-transmural MI are associated with more favourable outcomes.

Some have advocated the use of mechanical circulatory support to stabilise patients presenting with MI and shock. Dang and colleagues, ${ }^{6}$ in an analysis of 74 patients undergoing left ventricular assist device implantation for post-MI shock, showed that 1-year survival was higher in patients undergoing direct left ventricular assist device (LVAD) placement rather than revascularisation followed by LVAD implantation.

\subsection{CABG in Cardiogenic Shock}

In patients with acute MI complicated by cardiogenic shock, CABG has been shown to result in a reasonable survival rate. Earlier reports from the 1980s and 1990s suggested in-hospital mortality of approximately 10-30\% for those with cardiogenic shock undergoing CABG.

\footnotetext{
${ }^{4}$ Lee DC et al., J Thorac Cardiovasc Surg 2003.

${ }^{5}$ Thielmann et al., Ann Thorac Surg 2007.

${ }^{6}$ Dang NC et al., J Thorac Cardiovasc Surg 2005
} 
More recently, the SHOCK trial ${ }^{7}$ randomised 302 patients with acute MI and cardiogenic shock to medical therapy versus early revascularisation. Of the early revascularisation group, 38\% underwent $\mathrm{CABG}$ and 55\% PCI. One-year survival was improved for those receiving early revascularisation compared to medical therapy. However, the design of the trial meant that patients could receive PCI or CABG up to $54 \mathrm{~h}$ after symptom onset and still be considered to have received early revascularisation. Nevertheless, for patients with cardiogenic shock, CABG represents a reasonable strategy, despite the higher risk of post-operative low cardiac output and end-organ dysfunction.

\subsection{Peri-operative and Operative Considerations}

Compared to elective CABG, surgery in the context of AMI presents unique challenges for cardiac surgeons. The heightened risk of mortality and morbidity of emergency surgery compared to more conventional elective surgery must be carefully considered by surgeons and cardiologists when deciding upon the best mode of therapy.

\subsubsection{Antiplatelet Therapy}

Antiplatelet agents such as clopidogrel and ticagrelor are now commonly administered to patients in combination with aspirin as dual antiplatelet therapy (DAPT) at presentation with an acute coronary syndrome, or around the time of angiography and stent implantation to reduce the risk of stent thrombosis.

In urgent scenarios, or those in whom a stent has been placed to treat a culprit lesion, patients often require surgery while on dual antiplatelet therapy. The risk of peri-operative bleeding after CABG in patients receiving DAPT is substantially increased, with the risk of reoperation for ongoing bleeding being reported as being up to 6 times higher. A meta-analysis investigating timing of surgery in patients on clopidogrel found that patients on DAPT were more likely to have ongoing bleeding, require reoperation and experience adverse outcomes compared to those in whom a washout period of $>5$ days was observed. However, patients in whom a washout period of $>5$ days was observed, use of clopidogrel was associated with lower rates of mortality and myocardial infarction compared to those not on clopidogrel, suggesting a valuable role for DAPT in those presenting with an acute MI.

The American Heart Association/American College of Cardiology (AHA/ACC) and European Society of Cardiology/European Association of Cardiothoracic Surgery (ESC/EACTS) guidelines suggest cessation of clopidogrel or ticagrelor for at least 5 days prior to nonurgent CABG (Table 16.1). For urgent cases, clopidogrel or ticagrelor should be discontinued for at least $24 \mathrm{~h}$. At our centre, we generally cease clopidogrel or ticagrelor 3-5 days prior to $\mathrm{CABG}$.

\footnotetext{
${ }^{7}$ Hochman et al., N Eng J Med 1999
} 
Table 16.1 Summary of AHA and ESC recommendations

\begin{tabular}{|c|c|c|}
\hline Recommendation & $\begin{array}{l}2013 \\
\mathrm{AHA}^{\mathrm{a}}\end{array}$ & $\begin{array}{l}2017 \\
\text { ESC }^{\mathrm{b}}\end{array}$ \\
\hline $\begin{array}{l}\text { Urgent CABG is indicated in patients with STEMI and coronary } \\
\text { anatomy not amenable to PCI who have ongoing or recurrent ischaemia, } \\
\text { cardiogenic shock, severe HF or other high-risk features }\end{array}$ & Class I & \\
\hline $\begin{array}{l}\text { Immediate PCI is indicated for patients with cardiogenic shock if } \\
\text { coronary anatomy is suitable. If coronary anatomy is not suitable for } \\
\text { PCI, or PCI has failed, emergency CABG is recommended }\end{array}$ & & Class I \\
\hline $\begin{array}{l}\text { CABG should be considered in patients with ongoing ischaemia and } \\
\text { large areas of jeopardised myocardium if PCI of the ischaemia-related } \\
\text { artery cannot be performed }\end{array}$ & & Class IIa \\
\hline $\begin{array}{l}\text { CABG is recommended in patients with STEMI at time of operative } \\
\text { repair of mechanical defects }\end{array}$ & Class I & \\
\hline $\begin{array}{l}\text { The use of mechanical circulatory support is reasonable in patients with } \\
\text { STEMI who are haemodynamically unstable and require urgent CABG }\end{array}$ & $\begin{array}{l}\text { Class } \\
\text { IIa }\end{array}$ & Class IIb \\
\hline $\begin{array}{l}\text { Emergency CABG within } 6 \text { h of symptom onset may be considered in } \\
\text { patients with STEMI who do not have cardiogenic shock and are not } \\
\text { candidates for PCI or fibrinolytic therapy }\end{array}$ & $\begin{array}{l}\text { Class } \\
\text { IIa }\end{array}$ & \\
\hline $\begin{array}{l}\text { For nonurgent } \mathrm{CABG} \text {, discontinue clopidogrel and ticagrelor for at least } \\
5 \text { days before surgery and prasugrel for at least } 7 \text { days }\end{array}$ & Class I & $\begin{array}{l}\text { Class I } \\
(2014)^{\mathrm{c}}\end{array}$ \\
\hline $\begin{array}{l}\text { Clopidogrel or ticagrelor should be discontinued at least } 24 \mathrm{~h} \text { before } \\
\text { urgent on-pump CABG, if possible }\end{array}$ & Class I & $\begin{array}{l}\text { Class I } \\
(2014)\end{array}$ \\
\hline $\begin{array}{l}\text { The use of intra-aortic balloon pump (IABP) counter-pulsation can be } \\
\text { useful for patients with cardiogenic shock after STEMI }\end{array}$ & $\begin{array}{l}\text { Class } \\
\text { IIa }\end{array}$ & Class IIa \\
\hline
\end{tabular}

aO'Gara P et al., Circulation 2013

'Ibanez et al., Eur Heart J 2017

${ }^{c}$ Windecker S, Eur Heart J 2014

\subsection{Intra-aortic Balloon Pump (IABP)}

For patients who are haemodynamically unstable, the IABP is a useful adjunct when performing $\mathrm{CABG}$ in the context of acute MI. The IABP has been shown to decrease myocardial oxygen requirements. Earlier studies suggested a reduction in early mortality associated with IABP use. However, the IABP-SHOCK II trial, ${ }^{8}$ which randomised 600 patients with cardiogenic shock complicating acute MI to IABP or no IABP, showed that IABP therapy did not significantly reduce early mortality. The ACC/AHA guidelines have also changed the recommendation for IABP use in cardiogenic shock from a class I down to a class IIa recommendation.

At our centre, we generally insert an IABP for acute MI in patients with cardiogenic shock in preparation for transfer to the operating theatre so as to provide temporary stabilisation until cardiopulmonary bypass can be established.

\footnotetext{
${ }^{8}$ Thiele $\mathrm{H}$ et al., Lancet 2013.
} 


\subsection{Intra-operative Considerations}

In the last decade, observational and randomised studies have suggested a benefit in using additional arterial conduits (radial artery, right internal thoracic artery) to revascularise the myocardium in addition to the standard left internal thoracic artery to left anterior descending artery configuration. Indeed, the ACC/AHA guidelines give class II indications to the use of additional arterial conduits.

In general, the selection of conduits is similar to that of elective CABG cases. Unless patients are haemodynamically unstable, a LITA should still be harvested for revascularising the LAD territory, with additional supplemental conduits such as the long saphenous vein or radial artery.

Where patients are unstable with cardiogenic shock, it may be more prudent to cannulate the heart and establish cardiopulmonary bypass immediately following sternotomy, thus avoiding the 20-30 min delay associated with LITA harvest. Cardiopulmonary bypass with decompression of the heart substantially reduces wall tension and metabolic requirements. The LITA can then be harvested while on cardiopulmonary bypass. With unstable cases, we will generally harvest a LITA on cardiopulmonary bypass in younger patients with few comorbidities, while in older patients, we will use only peripheral conduits (radial artery, saphenous vein).

Both on-pump and off-pump CABG are acceptable techniques for surgery in acute MI. Fattouch et al. randomised 128 patients with STEMI to either on- or offpump CABG and found off-pump surgery to be associated with low mortality (1.6\%). Off-pump CABG was associated with lower rates of low cardiac output syndrome, mechanical ventilation, inotrope use and reoperation for bleeding. In our centre, on-pump CABG is our preferred technique; as such off-pump CABG in the acute setting is reserved for those cases where significant aortic calcification renders aortic cannulation and cross-clamping unsafe.

Some surgeons prefer to perform on-pump surgery without cardioplegic arrest (onpump beating heart $\mathrm{CABG}$ ) in the acute setting, as this has the potential to reduce the impact of ischaemic-reperfusion injury associated with aortic cross-clamping. The beating heart technique has been shown to achieve similar results compared to conventional CABG with comparable rates of early mortality and morbidity. ${ }^{9}$

\subsection{Results of CABG in Acute MI}

Earlier reports of CABG in acute MI showed mortality rates of 5-10\%. As thrombolysis and PCI have evolved to become first-line the therapies, the spectrum of patients presenting for surgery has changed significantly compared to patients included in those earlier reports. Contemporary reports of surgery in acute MI show early mortality rates of 5-20\%, with cardiogenic shock being a major predictor of early mortality. Long-term survival after emergency CABG is influenced chiefly by patient comorbidities and ventricular function.

${ }^{9}$ Chaudhry UA et al., Ann Thorac Surg 2015 


\subsection{Conclusions}

Bypass surgery, despite not being first-line, remains an important modality in the management of patients unsuccessful acute MI. It is reserved for cases where PCI is not feasible, or has been unsuccessful, and in those where revascularisation cannot be completed with PCI alone. The optimal time to operate on patients can be difficult to determine. In those with ongoing ischaemia, emergency surgery is indicated. However, in stable patients, delayed surgery is associated with improved outcomes. For cardiac surgeons, surgery for acute MI presents additional challenges including bleeding risk, conduit selection and the decision to utilise mechanical support. A collaborative "Heart Team" approach ensures the management approach is tailored specifically to the patient.

\subsection{Coronary Artery Bypass Surgery for STEMI}

William Y. Shi and Julian A. Smith

\subsection{Illustrative Cases}

When managing patients with acute MI, cardiologists and cardiac surgeons may be faced with a number of difficult clinical decisions. The two cases below represent "real-life" clinical cases from our institution and illustrate the key themes discussed in this chapter.

\subsubsection{Case 1: Delayed Surgery After PCI for Acute MI}

A 54-year-old man presented with an acute STEMI. He had experienced intermittent chest pain for several months. ECG revealed ST elevation in the inferior leads with ST depression in the anterolateral leads. He was loaded with $300 \mathrm{mg}$ aspirin and $180 \mathrm{mg}$ ticagrelor. He had no other past medical history. Coronary angiography revealed chronic total occlusion of the left anterior descending and left circumflex systems. The right coronary system was dominant. There was occlusion of the proximal segment with TIMI I flow due to acute plaque rupture. The right coronary artery was providing retrograde collateral blood supply to the left coronary system. The right coronary occlusion was deemed to be the culprit lesion, and this was stented using a drug-eluting stent (Fig. 16.1).

The patient was subsequently referred for consideration of surgery given the left coronary system was not suitable for PCI. Subsequent transthoracic echocardiogram demonstrated severe left ventricular dysfunction (LV ejection fraction $30 \%$ ). Troponin I peaked at 7500ng/L.

The patient experienced short episodes of chest pain upon return to the ward; as such he was commenced on heparin and glyceryl trinitrate infusion and thereafter 

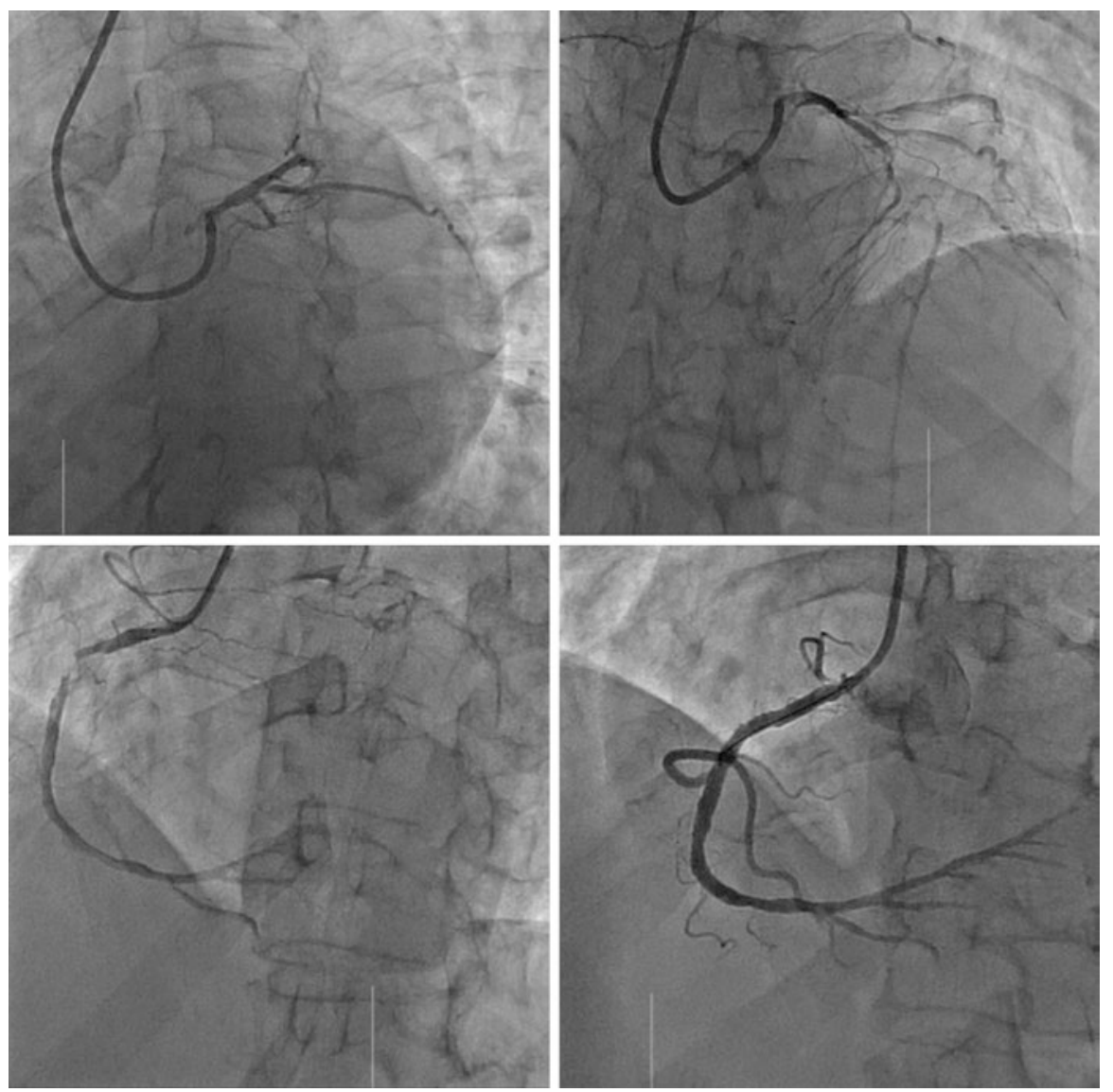

Fig. 16.1 Coronary angiogram demonstrating chronic occlusions of the LAD and circumflex vessels and acute occlusion of the proximal right coronary artery. Post-PCI result is demonstrated on the bottom right

remained pain-free. Given his haemodynamically stable state, poor ventricular function and substantial infarct size, the decision was made to delay surgery due to the high mortality risk associated with early surgery for acute MI. Due to recent PCI, dual antiplatelet therapy could not be ceased prior to surgery, and as such aspirin $100 \mathrm{mg}$ daily and ticagrelor $90 \mathrm{mg}$ twice daily were administered up until the day of surgery.

The patient subsequently underwent surgery for CABG 7 days later and received a left internal thoracic artery to his left anterior descending artery and a radial artery to an obtuse marginal branch of the circumflex. An epicardial left ventricular lead was placed to facilitate cardiac resynchronisation therapy should the patient require it in the future. An intra-aortic balloon pump was considered but ultimately not used as the patient was weaned off cardiopulmonary bypass on 
low-dose inotropes. Thromboelastography demonstrated abnormal platelet function, and platelet transfusion was required to obtain satisfactory haemostasis prior to surgical closure.

The patient was extubated on day 1 post-surgery. Aspirin was commenced on post-operative day 1, and ticagrelor was recommenced on post-operative day 2 . Recovery was complicated by post-operative atrial fibrillation for which he received amiodarone. The patient was ultimately discharged home on post-operative day 11 .

\subsubsection{Case 2: Emergency CABG for Ongoing Ischaemia}

A 72-year-old man presented with an inferior ST-elevation myocardial infarction. He had a past history of type 2 diabetes mellitus, hypercholesterolaemia, chronic obstructive pulmonary disease, obesity and chronic renal impairment. He presented with several hours of chest pain with inferior ST elevation. He developed cardiogenic shock and acute pulmonary oedema requiring an adrenaline infusion and mechanical ventilation before transfer to the cardiac catheterisation laboratory.

Coronary angiography demonstrated an occluded distal right coronary artery with evidence of thrombus. His left anterior descending artery also showed severe disease with mid-segment occlusion (Fig. 16.2). Attempted PCI to the right coronary lesion was unsuccessful due to inability to pass the guidewire beyond the lesion. There was ongoing inferior ST elevation and hypotension. The patient was referred for emergency CABG. Given the patient's haemodynamic instability, an IABP was inserted in the catheterisation laboratory prior to transfer to the operating theatre. Transthoracic echocardiography demonstrated severe left ventricular impairment (EF 15\%).

In the operating theatre, after sternotomy, cardiopulmonary bypass was immediately established. Simultaneously, the long saphenous vein was harvested. The LITA was not harvested. Saphenous vein was used to revascularise the LAD and the PDA. The patient was weaned off cardiopulmonary bypass using the IABP as an adjunct.
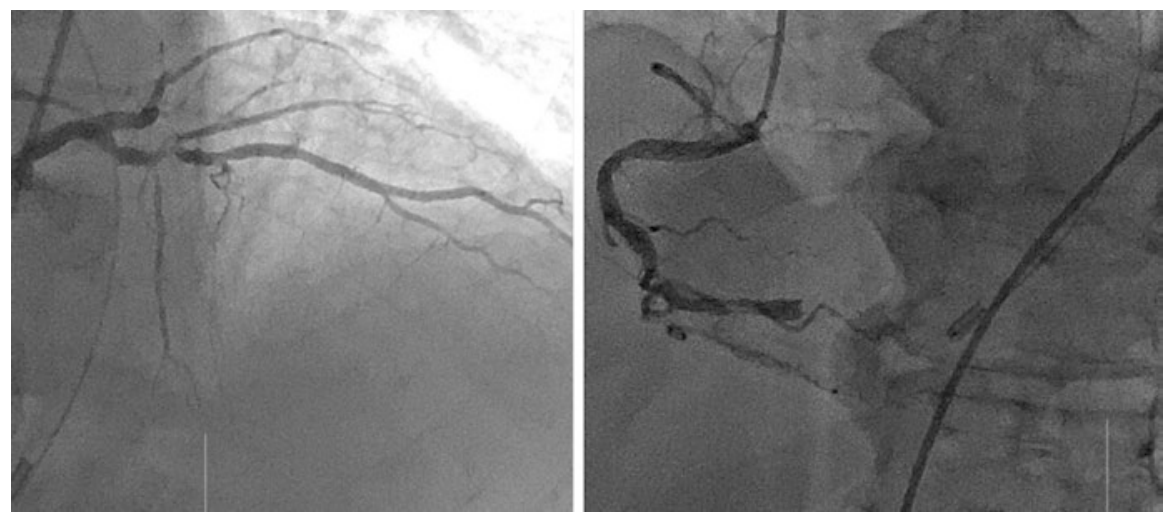

Fig. 16.2 Coronary angiogram of case 2, demonstrating occluded LAD and RCA. The RCA had evidence of thrombus formation, suggesting its role as the culprit lesion 
Post-operatively, the patient was supported on adrenaline infusion. The IABP was weaned and removed on post-operative day 2 . The patient's recovery was complicated by prolonged ventilation, requiring tracheostomy and renal failure, requiring temporary dialysis. The patient was discharged after 1 month to a rehabilitation facility and then subsequently discharged home.

\section{Further Readings}

Ibanez B, James S, Agewall S, et al. 2017 ESC Guidelines for the management of acute myocardial infarction in patients presenting with ST-segment elevation: The Task Force for the management of acute myocardial infarction in patients presenting with ST-segment elevation of the European Society of Cardiology (ESC). Eur Heart J. 2017;39(2):119-77. https://doi. org/10.1093/eurheartj/ehx393.

Lee DC, Oz MC, Weinberg AD, Ting W. Appropriate timing of surgical intervention after transmural acute myocardial infarction. J Thorac Cardiovasc Surg. 2003;125:115-9.

Mohr FW, Morice MC, Kappetein AP, et al. Coronary artery bypass graft surgery versus percutaneous coronary intervention in patients with three-vessel disease and left main coronary disease: 5-year follow-up of the randomised, clinical SYNTAX trial. Lancet. 2013;381:629-38.

O'Gara PT, Kushner FG, Ascheim DD, et al. 2013 ACCF/AHA guideline for the management of ST-elevation myocardial infarction: a report of the American College of Cardiology Foundation/American Heart Association Task Force on Practice Guidelines. Circulation. 2013;127:e362-425.

Thiele H, Zeymer U, Neumann FJ, et al. Intra-aortic balloon counterpulsation in acute myocardial infarction complicated by cardiogenic shock (IABP-SHOCK II): final 12 month results of a randomised, open-label trial. Lancet. 2013;382:1638-45.

Open Access This chapter is licensed under the terms of the Creative Commons Attribution 4.0 International License (http://creativecommons.org/licenses/by/4.0/), which permits use, sharing, adaptation, distribution and reproduction in any medium or format, as long as you give appropriate credit to the original author(s) and the source, provide a link to the Creative Commons license and indicate if changes were made.

The images or other third party material in this chapter are included in the chapter's Creative Commons license, unless indicated otherwise in a credit line to the material. If material is not included in the chapter's Creative Commons license and your intended use is not permitted by statutory regulation or exceeds the permitted use, you will need to obtain permission directly from the copyright holder. 